\title{
Designing Good LDPC Codes for Markov-Modulated Channels
}

\author{
Andrew W. Eckford \\ Dept. of Electrical Engineering \\ University of Notre Dame \\ 275 Fitzpatrick \\ Notre Dame, Indiana \\ 46556 USA \\ E-mail: aeckford@nd.edu
}

\author{
Frank R. Kschischang \\ The E. S. Rogers Sr. Dept. of ECE \\ University of Toronto \\ 10 King's College Road \\ Toronto, Ontario \\ M5S 3G4 Canada \\ E-mail: frank@comm.utoronto.ca
}

\author{
Subbarayan Pasupathy \\ The E. S. Rogers Sr. Dept. of ECE \\ University of Toronto \\ 10 King's College Road \\ Toronto, Ontario \\ M5S 3G4 Canada \\ E-mail: pas@comm.utoronto.ca
}

\begin{abstract}
We present a reduced-complexity approximate density evolution scheme that is particularly suitable for Markov-modulated channels, based on the semi-Gaussian approximation from [1]. We propose a design algorithm whose complexity is comparable to the memoryless case, assuming unlimited precomputation is allowed. We also present degree sequences representing some of the best known codes in the GE channel which were designed using this technique. This design tool can be easily extended to more complicated Markov-modulated channels.
\end{abstract}

\section{MARKOV-MODUlated CHANNELS}

The Markov-modulated channel is a channel model that generalizes memoryless channels by making the channel parameter a function of the state of a hidden Markov chain. Formally, suppose the channel transition function is written $f_{Y}(y \mid x, s)$ with some hidden channel state $s$, and suppose $s$ is an element of a Markov chain. Consider a Markov chain of length $n$, with a hidden vector $\mathbf{s} \in \mathcal{S}^{n+1}$ of channel states, a hidden vector $\mathbf{x} \in\{0,1\}^{n}$ of transmitted symbols, and a vector $\mathbf{y} \in \mathcal{Y}^{n}$ of observations. Given a state transition probability matrix $\mathbf{P}$, the PMF of the channel state process can be written

$$
\operatorname{Pr}(\mathbf{s})=\operatorname{Pr}\left(s_{1}\right) \prod_{i=1}^{n} \operatorname{Pr}\left(s_{i+1} \mid s_{i}\right),
$$

where the values of $\operatorname{Pr}\left(s_{i+1} \mid s_{i}\right)$ are obtained from $\mathbf{P}$. Furthermore, a channel use occurs at each time instant, and the channel state affects the channel output probability through a conditional PDF $f_{Y_{i}}\left(y_{i} \mid x_{i}, s_{i}\right)$ for each $s_{i} \in \mathcal{S}$. Thus, we may write

$$
\operatorname{Pr}(\mathbf{y}, \mathbf{s} \mid \mathbf{x})=\operatorname{Pr}\left(s_{1}\right) \prod_{i=1}^{n} \operatorname{Pr}\left(s_{i+1} \mid s_{i}\right) f_{Y_{i}}\left(y_{i} \mid x_{i}, s_{i}\right) .
$$

The prior density for the channel inputs $\mathbf{x}$ may be provided by the indicator function of an LDPC code. Representation of such a system on a factor graph and estimation-decoding algorithms are well known (for example, see [2]).

\section{REDUCED-COMPLEXITY DESIGN TOOL}

Typically, approximate DE techniques will replace the exact density with a member of a family indexed by a single parameter, $\nu$. The approximation of DE for LDPC decoding in Markov channels is complicated by the requirement to account for channel estimation, which is usually dependent on iteration number, rather than the input extrinsic message.

Consider the use of a message-passing schedule in which one sub-iteration of LDPC decoding is followed by $N$ subiterations of channel estimation, where $N$ is very large. In the limit as the number of iterations approaches $\infty$, we can show that this schedule has the same performance as the standard parallel-update schedule. In this case, we have observed that whenever the same extrinsic message density is applied over a large number of iterations, a stable channel message density will result, regardless of the initial channel message density.

Using this observation, suppose that the extrinsic information densities are replaced by their single-parameter approximations, parameterized by $\nu$. For each value of $\nu$, a stable channel density can be obtained by applying a large number of operations through the GE subgraph, using the approximate extrinsic density corresponding to that value of $\nu$. The resulting stable channel densities form a family of channel densities parameterized by $\nu$.

Briefly, the design algorithm proceeds as follows:

- Precalculation: For each value of $\nu$ and some sufficiently large $N$, use density evolution or Monte Carlo methods to obtain the stable channel message density corresponding to $\nu$.

- Semi-Gaussian approximation: Fix the check degree sequence $\rho$. For each variable degree $i$, use density evolution to obtain a transfer function $\nu_{\text {out }}=f_{i}\left(\nu_{\text {in }}\right)$. For each $\nu_{\mathrm{in}}$, the channel density is selected as the appropriate entry from the precalculated family. See [1] for more details.

- Optimization: The functions $f_{i}(\cdot)$ may be combined linearly to obtain the overall transfer function $f\left(\nu_{\text {in }}\right)=$ $\sum_{i} \lambda_{i} f_{i}\left(\nu_{\mathrm{in}}\right)$. Successful decoding is achieved as long as $f\left(\nu_{\text {in }}\right)<\nu_{\text {in }}$ everywhere. Linear programming may be used to maximize rate subject to successful decoding.

We implemented this design tool in the two-state GilbertElliott (GE) channel. In [3], a rate $1 / 2$ Turbo code achieved $P_{\text {err }}=10^{-6}$ in a $\mathrm{GE}$ channel with inversion probabilities $\left(\eta_{B}, \eta_{G}\right)=(0.5,0.0192)$ and state transition probabilities $\left(\operatorname{Pr}\left(s_{i+1}=B \mid s_{i}=G\right), \operatorname{Pr}\left(s_{i+1}=G \mid s_{i}=B\right)\right)=$ $(0.0044,0.0156)$. Our design tool found an LDPC code with rate 0.596 and degree sequence $\lambda_{2}=0.25, \lambda_{3}=0.6209$, $\lambda_{6}=0.1291 ; \rho_{7}=1$ with the same performance in this channel.

\section{REFERENCES}

[1] M. Ardakani and F. R. Kschischang, "A More Accurate OneDimensional Analysis and Design of LDPC Codes," submitted to IEEE Trans. Commun.

[2] A. W. Eckford, Low-Density Parity-Check Codes for GilbertElliott and Markov-Modulated Channels, Ph.D. thesis, University of Toronto, 2004.

[3] J. Garcia-Frias and J. D. Villasenor, "Turbo decoding of Gilbert-Elliott channels," IEEE Trans. Commun., vol. 50, no. 3, pp. 357-363, Mar. 2002. 